\title{
ENHANCEMENT OF DISSOLUTION RATE OF POORLY SOLUBLE DRUG ITRACONAZOLE BY NANOSUSPENSION TECHNOLOGY: ITS PREPARATION AND EVALUATION STUDIES
}

\author{
RAJAKUMAR DEVARA ${ }^{1}$, MOHAMMED HABIBUDDIN ${ }^{2}$, JITHAN AUKUNURU ${ }^{3,4 *}$ \\ ${ }^{1}$ Department of Mother Teresa College of Pharmacy, Ghatkesar, Hyderabad, India. ${ }^{2}$ Department of Shadan College of Pharmacy, \\ Peerancheru, Hyderabad, India. ${ }^{3}$ Department of Mankind Research Center, Manesar, Haryana, India. ${ }^{4}$ Technology Consultants, \\ Hyderabad, India. Email: aukunjv@gmail.com
}

Received: 15 May 2017, Revised and Accepted: 15 July 2017

ABSTRACT

Objective: The objective of this study was to prepare and evaluate itraconazole (ITZ) nanosuspensionsusing polymer Eudragit RL-100 and stabilizer Tween- 80 by nanoprecipitation method.

Materials and Methods: Itraconazole is a potent broad-spectrum Biopharmaceutical Classification System Class II triazole antifungal drug. Nanosuspensions were prepared using solvent displacement/nanoprecipitation method with the help of Eudragit RL-100 as rate-controlled polymer in different ratios and using Tween-80 as stabilizer. The nanosuspension preparation was optimized for particle size by investigating two factors that are solvent:anti-solvent ratio and surfactant concentration, at three levels. The prepared nanosuspensions were evaluated and characterized for particle size, drug excipient compatibility, percentage yield, drug entrapment efficiency, surface morphology, zeta potential, saturation solubility, solid state, and in vitro drug release studies.

Results: The nanosuspensions of itraconazole were successfully prepared using solvent displacement/nanoprecipitation method. The two factors solvent: anti-solvent ratio and surfactant concentration influenced the particle size of the nanosuspensions prepared. The Fourier-transform infrared spectroscopy studies confirmed that drug and excipients are compatible, and the X-ray powdered diffraction and differential scanning calorimetry results indicated that the nanoprecipitation method led to the amorphization of itraconazole. Itraconazolenanosuspensions increased the saturation solubility to an extent of 4 times. Itraconazole nanosuspensions completely dissolved in the dissolution medium within $10 \mathrm{~s}$ and $72 \%$ drug release within $5 \mathrm{~min}$, while the pure drug was dissolved only up to $20 \%$ in $15 \mathrm{~min}$ and nanosuspensions showed increased dissolution rate of 3 folds, the active drug.

Conclusions: Stable itraconazole nanosuspensions were successfully prepared and these nanosuspensions demonstrated dramatic improvement in dissolution rate of the active drug.

Keywords: Itraconazole, Eudragit RL-100, Tween 80, Nanoprecipitation/solvent displacement, Dissolution rate.

(c) 2018 The Authors. Published by Innovare Academic Sciences Pvt Ltd. This is an open access article under the CC BY license (http://creativecommons. org/licenses/by/4. 0/) DOI: http://dx.doi.org/10.22159/ajpcr.2018.v11i4.19933

\section{INTRODUCTION}

Nanotechnology and related technologies have enhanced the productivity of formulation development technology tremendously with a wide range of applications [1]. Poor water-soluble compounds are difficult to develop as drug products using conventional formulation techniques and are frequently abandoned early in discovery. Solubility is an essential factor for drug effectiveness, independent of administration route. It also poses a major challenge for pharmaceutical companies developing new pharmaceutical products, since nearly half the active substances being identified through the new paradigm in high-throughput screening are either insoluble or poorly soluble in water [2-6]. Nanotechnology-based formulations have been successful in solving this problem which has been troubling pharmaceutical industry for a long time [4,7-9]. Oral drug delivery continues to be the preferred gateway of a drug into the bloodstream, especially when repeated or routine administration is necessary. However, for effective delivery through the oral route, a therapeutic agent must first dissolve in the gastrointestinal lumen [10]. This represents a major challenge in the field of pharmaceutical drug formulation, namely, the design of an oral dosage form for poorly soluble drugs under biopharmaceutical classification system (BCS) Class-II and highly lipophilic drugs that would enhance their notoriously poor bioavailability. Due to their low bioavailability, several potential drugs have to be delayed or abandoned in pharmacological screenings because of their lipophilicity. Their dissolution rate is the limiting factor in the most case because drugs with high lipophilicity can permeate bio-membranes quickly. In general, it can be stated that the rate of absorption affects the onset and extent of the clinical effect and is determined by the dissolution of the drug and the subsequent transport over the intestinal membrane and passage of the liver, and these two aspects form the basis of the BCS which is incorporated in the guidelines of the Food and Drug Administration (FDA). Drugs can be categorized into four biopharmaceutical classes based on solubility and permeability, and this system supports the definition of the role of in vitro dissolution and role of correlation or non-correlation with in vivo process which can also be explained from this classification system. According to BCS which is incorporated in the guidelines of the FDA, Class II drugs are with high permeability and low solubility and have a high absorption number but a low dissolution number. The bioavailability of a BCS class II drug is rate-limited by its dissolution so that even a small increase in dissolution rate sometimes results in a large increase in bioavailability. Therefore, an enhancement of the dissolution rate of the drug is thought to be a key factor for improving the bioavailability of BCS Class II drugs. In this class of drugs, dissolution profile must be clearly defined and reproducible, since drug dissolution is rate controlling step in in vivo drug absorption. The absorption for Class II drugs is usually slower than class I and occurs over a longer period, and IVIVC is usually expected for Class II drugs [11,12]. Given the increasing number of compounds emerging from discovery programs having poor 
solubility and/or dissolution rate, pharmaceutical scientists are constantly seeking new formulation approaches to obtain an adequate oral bioavailability. The effort to improve the dissolution and solubility of a poorly and practically water-insoluble drugs remains one of the most challenging tasks in drug development. Currently, novel possibilities are offered by the rapidly emerging field of nanoscience, and one among them that has rapidly gained a proven record within the pharmaceutical sciences is the formulation of drugs as drug nanocrystals or nanosuspensions $[13,14]$. A pharmaceutical nanosuspension is a submicron colloidal dispersion of nanosized drug particles that are produced by a suitable method and stabilized by a suitable stabilizer. Formulation of stable nanosuspension requires a careful selection of stabilizers which are needed to stabilize the nanoparticles against interparticle forces and prevent them from aggregating and agglomeration. The main function of a stabilizer is to wet the drug particles thoroughly and to prevent Ostwald's ripening and agglomeration of nanosuspension to yield a physically stable formulation by providing steric or ionic barrier. Nanosuspensions are very finely dispersed solid drug particles in an aqueous vehicle for either oral and topical use or parenteral and pulmonary administration. The particle size distribution of the solid particles in nanosuspension is usually $<1 \mu$ with an average particle size ranging between 200 and $600 \mathrm{~nm}$ having a size below $1 \mu \mathrm{m}$. Nanosized particles can increase solution velocity and saturation solubility because of the vapor pressure effect. In addition, the diffusional distance on the surface of drug nanoparticles is decreased, thus leading to an increased concentration gradient. The increases in surface area and concentration gradient lead to a much more pronounced increase in the dissolution velocity as compared to a micronized product. Furthermore, the saturation solubility is increased as well. Another possible explanation for the increased saturation solubility is the creation of high energy surfaces when disrupting the more or less ideal drug microcrystals to nanoparticles. In the first-generation applying disintegration techniques, this was achieved by pearl milling leading to the product nanocrystals. This patent protected technology was developed by Liversidge to produce nanocrystals, the drug powder is dispersed in a surfactant solution, and the obtained suspension undergoes a pearl milling process for hours up to several days. The second-generation products are drug nanoparticles produced by high-pressure homogenization leading to the so-called nanosuspensions [15-17]. The nanosuspensions were developed by Muller et al. in 1994 and the patent rights owned by drug delivery services $\mathrm{GmbH}$ in Germany and now owned by SkyePharma PLC. Drug nanocrystals can be obtained either by particle size reduction (top-down approach) or by building up particles from a solution (bottom-up approach). Bottom-up techniques can be considered as the oldest techniques to prepare drug nanocrystals based on precipitation techniques, also known as "via humidaparatum" (v.h.p.). Bottom-up is a precipitation method, building up nanoparticles from drug molecules through precipitation by addition of anti-solvent. In bottom-up technology, the drug is dissolved in a solvent, which is then added to non-solvent to precipitate the crystals. Sucker et al. used a precipitation technique to produce nanoparticles by dissolving the poorly soluble drug (API) in a solvent and adding the solvent to a nonsolvent (anti-solvent) that causes precipitation of the fine drug particle in the presence of a stabilizer. The conventional methods of precipitation (hydrosols) are called bottom-up technology. Within the group of the top-down techniques, media milling is the most popular technology, and 4 of the 5 currently marketed products rely on this technology such as Rapamune, Emend, TriCor, and Megace ES which are produced by media milling, whereas high-pressure homogenization is used for Triglide [18,19]. During media milling, nanosuspensions are produced by grinding a suspension of the drug and a suitable stabilizer in water with milling media such as yttrium-stabilized zirconium beads. Nanoparticles are usually produced in liquid media, thereby forming a nanosuspension. There is, however, a general preference for solid oral dosage forms over liquid forms, for patient convenience and physical stability reasons. As the primary objective of a nanoparticulate system

is rapid dissolution, disintegration of the solid form and redispersion of the individual nanoparticles should be equally rapid so that it does not impose a barrier on the overall dissolution process. Solidification methods for this transformation include pelletization, granulation, spray drying, and lyophilization and transformed into solid dosage form. Before solidification, a matrix-former is often added to the suspension for drying are water-soluble sugars such as sucrose, lactose and mannitol, as adapted from freeze-drying. There are several physical and chemical factors that play a major role in the instability of prepared nanoparticles. The overall stability studies are important to maintain nanosuspension physical and chemical stability [20-22]. Itraconazole is

a antifungal agent which is a weak base soluble in lipids. It is a BCS class II drug. It is soluble in acidic $\mathrm{pH}$, and thus, gastric acidity is required for adequate dissolution. The solubility in $0.1 \mathrm{~N} \mathrm{HCl}$ is approximately $4-6 \mathrm{mcg} / \mathrm{ml}$, and in water, it is $1-4$ $\mathrm{ng} / \mathrm{ml}$. Itraconazole exhibits very poor oral bioavailability owing to its insolubility in intestinal fluids. Its oral bioavailability is increased when taken with food compared to when taken in

fasting condition. Thus, oral itraconazole is prescribed to be taken after meals. This could be because of its interactions of the drug with food, physicochemical conditions of the gastrointestinal tract, higher dissolution in the presence of food, and not reaching the saturation solubility at acidic $\mathrm{pH}$ in the stomach in the fasting state. An itraconozole tablet when taken with food and without food is the need of the hour, and there should not be any fluctuations in the bioavailability of the drug in both the conditions. We hypothesize that if the solubility of this drug is increased along with the dissolution rate using technology such as nanosuspension technology, a formulation that can be taken in any condition would have the same oral bioavailability. Further, nanosuspension technology when developed as amorphous nanosuspension if the stability is resolved, the saturation solubility can be enhanced several times, which itself can increase the oral bioavailability. Therefore, there could not be any change in the oral bioavailability of itraconazole with and without food. Therefore, the objective of this study was to develop itraconazole nanosuspension and characterize it for various in vitro properties [23].

\section{MATERIALS AND METHODS}

Itraconazole is a gift sample from Nosch labs Pvt., Ltd., Eudragit RL 100, Tween 80, dichloromethane, and acetone are from SD fine chemicals which are AR grade. The pre-formulation studies with the itraconozole obtained were performed using conventional and reported techniques. The ultraviolet (UV)-visible spectrum, solubility, flow properties, and drug crystallinity were determined.

\section{Preparation of itraconazole nanosuspensions}

Several batches of itraconazole nanosuspensions were formulated by nanoprecipitation/solvent displacement method using probe sonication. Required quantity of itraconazole was weighed and dissolved in appropriate quantity of dichloromethane. Dichloromethane was used as a solvent, Eudragit RL 100 as polymer, and Tween 80 as

Table 1: Preparation of itraconazole nansuspension by nanoprecipitation/solvent displacement method

\begin{tabular}{llllllll}
\hline S.No & Formulation & F 1 & F 2 & F 3 & F 4 & F 5 & F 6 \\
\hline 1 & Itraconazole & 100 & 100 & 100 & 100 & 100 & 100 \\
2 & Eudragit RL 100 (mg) & 250 & 250 & 500 & 500 & 1000 & 1000 \\
3 & Tween 80 (mL) & 1.0 & 1.5 & 1.0 & 1.5 & 1.0 & 1.5 \\
4 & Dichloromethane (mL) & 10 & 10 & 10 & 10 & 10 & 10 \\
5 & Distilled water (mL) & 20 & 20 & 20 & 20 & 25 & $1: 2.5$ \\
6 & Solvent: anti-solvent ratio & $1: 2$ & $1: 2$ & $1: 2$ & $1: 2$ & $1: 2.5$ \\
\hline
\end{tabular}


a surfactant to stabilize the nanosuspension formulation. Eudragit RL 100 is dissolved in dichoromethanol, and different concentrations of Tween $80(1 \%)$ in required quantities of distilled water were dissolved in a $250 \mathrm{ml}$ Buchner flask given in Table 1. Dissolved dug solution is added to polymer-surfactant solution drop wise with a constant stirring on a magnetic stirrer at $8000 \mathrm{rpm}$ using a butterfly syringe for 3-4 h. Here, the effect of polymer, stabilizer, and solventanti-solvent ratio was studied. Vaccum was applied by placing paper on Buchner flask while stirring till the solvent evaporates and finally subjected to sonication by Probe Sonicator (Q-sonica, LLC, USA) for 5 min to prepare nanosuspensions. Product was filtered and dried at room temperature in a dessicatorover night. Finally, the product was filtered and centrifuged (Hittich, Zentrifugen, Model-1195 a, Mikro 220R, Germany) at $7000 \mathrm{rpm}$ for $10 \mathrm{~min}$. All the drug nanosuspensions were freeze dried using Freeze Dryer (Mini Lyodel, Delvac Pumps Pvt., Ltd., Chennai, India). The drug nanosuspension powder freeze dried into free flowing powder. In this study, a freezing rate gradient was used to systematically investigate the effect of cryoprotectants on the redispersibility of nanoparticles as a function of freezing rate in lyophilization. Sucrose was used as cryoprotectant for nanosuspension, and fast freezing rate and a high cryoprotectant concentration were generally favored $[24,25]$.

Preformulation studies as investigation of physical and chemical properties of drug substance alone or in combination with other excipient, which could affect drug performance and development of an efficacious dosage form. It acts as a foundation for developing good formulations and successful commercial products, to increase drug stability, to improve drug bioavailability, to reduce drug-excipient incompatibility, and to determine its kinetic rate profile.

Characterization and evaluation of nanosuspensions [26,27]

Percentage yield

To determine the yield, the weight of nanosuspensions obtained at the end of preparation was determined. The total weight of raw materials used to obtain this nanosuspension was determined to obtain the theoretical yield.

Percentage yield was then determined using the following formula:

Percentage yield $=($ Practical yield $/$ theoretical yield $) \times 100$

\section{Drug encapsulation efficiency (EE)}

To determine the EE, protocol developed in our laboratory was used. The amount of drug entrapped was estimated by dissolving the $100 \mathrm{mg}$ of nanosuspension in methonal and then sonicated under vigorous shaking for $1 \mathrm{~h}$. The resultant solution was centrifuged. The drug content in supernatant solution was analyzed spectrophotometrically using UVvisible spectrophotometer (Merck, Thermo Scientific Evolution 201) at $260 \mathrm{~nm}$ for drug itraconazole with further dilutions against appropriate blank. The amount of the drug entrapped in the nanosuspensions was calculated using the following formula:

Drug entrapment efficiency $(\%)=$ Amount of drug actually present in the supernatant/Theoretical drug load expected $\times 100$

\section{Particle size analysis}

The mean particle size was determined using optical microscopy method. In this method, the size of 250 particles was determined and the average particle size was calculated. Optical microscope can detect particles of sizes $>600 \mathrm{~nm}$ with accuracy. If particles produced are in this size range, this technique can be conveniently used to measure the particle size.

\section{Scanning electron microscopy (SEM)}

To examine the particle surface morphology and shape, SEM was used. A concentrated aqueous suspension was spread over a slab and dried under vacuum. The sample was shadowed in a cathodic evaporator with gold layer $20 \mathrm{~nm}$ thick. Photographs were taken using a Scanning Electron Microscope (Hitachi, S-3700N, Tokyo, Japan) operated at
$20 \mathrm{kV}$. The smallest size nanosupension was used for determining surface morphology.

\section{Zeta potential}

The zeta potential is used to predict the storage stability of colloid dispersed systems during the shelf storage, and it reflects the electrostatic barriers, which would prevent nanosuspension from aggregation and agglomeration. Particle aggregation will also likely to occur if particles had low zeta potential. In general, zeta potential of particles should be at least $-30 \mathrm{mV}$ for electrostatically stabilized systems or $-20 \mathrm{mV}$ for sterically stabilized systems to obtain a physically stable nanosuspension. The zeta potential was used to measure the electric charge at the surface of the particles, indicating the physical stability of colloidal systems. In this study, the zeta potential was assessed by determining the electrophoretic mobility of the particles. The zeta potential was measured using a nanoparticle analyzer and zeta potential measurement and zetasizer (Horiba SZ-100, Malvern Instruments, U.K). Samples were diluted with the respective original dispersion medium which provides information regarding the thickness of the diffuse layer. Diluted nanosuspension was added to the sample cell (quartz cuvette) and was put into the sample holder unit, and zeta potential was measured.

\section{Saturation solubility}

The saturation solubility of the particles is essential as it affects the bioavailability of the drug, the rate of drug release into dissolution medium, and consequently, the therapeutic efficiency of the pharmaceutical product. Saturation solubility is defined as the maximum quantity of a compound (solute) that can be dissolved in a certain quantity of a specific solvent at a specified temperature. Saturation solubility of pure drugs and nanosuspensions in different non-volatile solvents was estimated by conducting saturation solubility studies. The saturation solubility of the prepared nanosuspension and the pure drug were determined using rotary shaker (Neo labs instruments, Mumbai). Excess amount of drug was added to $10 \mathrm{ml}$ of solvent and $1.5 \mathrm{ml}$ of nanosuspension in different vials and subjected to continuous stirring using rotary shaker for $72 \mathrm{~h}$ at $25^{\circ} \mathrm{C}$ in water. Then, $1.5 \mathrm{ml}$ of nanosuspension was filled into centrifugation tube and centrifuged at $10000 \mathrm{rpm}$ for $30 \mathrm{~min}$ with cooling centrifuge (Hittich, Zentrifugen, Model-1195 a, Mikro 220R, Germany). Supernatant was filtered and analyzed spectrophotometrically using UV-visible spectrophotometer (Merck, Thermo Scientific Evolution 201) at $260 \mathrm{~nm}$ for respective itraconazole drug. The UV method for drug assay in saturation solubility and dissolution studies was developed in our laboratory and further validated. Linearity was achieved over a range of $100 \mathrm{ng} / \mathrm{ml}-10 \mu \mathrm{g} / \mathrm{ml}$. This assay was conveniently used to determine the drug samples.

\section{Drug-excipient compatibility studies (Fourier-transform infrared spectroscopy [FTIR] studies)}

FTIR spectrum of drug, polymer, and physical mixture of drug with polymers and optimized formulation was obtained on FTIR instrument. Sample about $5 \mathrm{mg}$ was mixed thoroughly with $100 \mathrm{mg}$ potassium bromide IR powder and compacted under vacuum at a pressure of about 12 Psi for $3 \mathrm{~min}$. The resultant disc was mounted in a suitable holder in IR spectrophotometer (Shimadzu 8400S, Tokyo, Japan), and the spectrum was scanned over the wave number range of 4000$400 \mathrm{~cm}^{-1}$ in a scan time of $12 \mathrm{~min}$. IR helps to confirm the identity of the drug and to detect the interaction of the drug with the carriers.

\section{Solid-state studies/properties}

\section{$X$-ray powder diffraction analysis}

$\mathrm{X}$-ray powdered diffraction was used to investigate the physical nature of the encapsulated drug on fabrication and variety of transformations during pharmaceutical processing and storage such as polymorphic transformations, alterations in crystallinity, changes in state, and degree of hydration. The drug crystalline state in the polymer sample was evaluated by powder X-ray diffraction analysis. X-ray spectra were recorded with X Pert-PRO multipurpose X-ray diffractometer 
(Shimadzu XRD-7000, Tokyo, Japan) using Ni-filtered, Cu Ka radiation, a voltage of $45 \mathrm{kV}$, and a current of $40 \mathrm{~mA}$ with a scintillation counter. The instrument was operated in the continuous scanning speed of $4^{\circ} / \mathrm{min}$ over a $2 \theta$ range of $5^{\circ}-40^{\circ}$. The samples were grinded using a Wedgewood mortar and pestle, placed into the cavity of an aluminum sample holder, and packed smoothly using a glass slide. The smallest size nanosuspension was used in the study.

\section{Differential scanning calorimetry (DSC)}

DSC studies were performed to understand the nature of the encapsulated drug in the polymer, and the physical state of drug in the polymer matrix would also influence its release characteristics. To probe this effect, DSC analysis was performed. Thermal analysis and properties of the powder samples such as itraconazole and optimized nanosuspension were investigated with a DSC (Shimadzu DSC 60, Tokyo, Japan). Approximately $10 \mathrm{mg}$ of sample was analyzed in an open aluminum pan and heated at scanning rate of $10^{\circ} \mathrm{C} / \mathrm{min}$ between $0^{\circ} \mathrm{C}$ and $400^{\circ} \mathrm{C}$ under nitrogen atmosphere. Magnesia was used as the standard reference material to identify physical changes of drug in the formulation. Hence, it indicates the physical nature of drug which is changed in the formulation from crystalline to amorphous.

\section{In vitro drug release studies}

In vitro dissolution studies of samples were carried out using USP XIV (Electro lab TDT-082, Model-ETC 11L, Mumbai, India) apparatus II paddle method by dispersed powder technique. Accurately weighed samples were added to $500 \mathrm{ml}$ of buffer media simulated gastric fluid without pepsin at $\mathrm{pH} 1.2 \mathrm{or} \mathrm{pH} 1.2$ acetate buffer at $37 \pm 0.5^{\circ} \mathrm{C}$ and stirred at $50 \mathrm{rpm}$. An aliquot of $10 \mathrm{ml}$ was withdrawn at different time intervals. The solid particles were prevented from pipetting by withdrawing the sample through a pipette fitted with a cotton plug. An equal volume of fresh dissolution medium was immediately replaced. The filtered samples were assayed spectrophotometrically at $260 \mathrm{~nm}$, respectively, for itraconazole drug. The dissolution of nanosuspension was compared with the dissolution of equivalent amount of the pure drug itraconazole.

\section{RESULTS AND DISCUSSION}

Preformulation studies for itraconazole have been performed to know the drug physical properties so as to design it to a suitable formulation. Itraconazole is a BSC Class II drug with low solubility and high permeability. Itraconazole is soluble in organic solvents such as dichloromethane and dimethylformamide. Water solubility was found to be very less $1-4 \mathrm{ng} / \mathrm{ml}$. Drug is crystalline in nature. Particle size reduction is done by nanosuspension method which is a novel technique to increase saturation solubility and dramatic increase in dissolution rate. Standard graph for itraconazole has been prepared by calibration curve method and scanned for the $\lambda_{\max }$ and found to be $260 \mathrm{~nm}$. Different batches of itraconazole nanosuspensions were prepared using Eudragit RL 100 polymer and Tween-80 surfactant concentration to obtain nanoparticles with a reduced particle size. Considering the percentage yield of $85.2 \%$ and drug EE $87.32 \%$ and reduction in particle size of $0.86 \mu \mathrm{m}$, batch F6 was preferred to be the best among all the batches. It has been observed that the particle size was reduced for the batch 6 with 1:1.25 solvent:anti-solvent ratio with $1.5 \mathrm{ml}$ of surfactant concentration with particle size $0.86 \mu$ and concentration of drug to Eudragit 1:10 with high yield of drug. The average particle size was found to be $0.86-1.22 \mu \mathrm{m}$. Reduction in the particle size of tonanometer range led to the increase in saturation solubility of nanosuspensions. The saturation solubility for pure drug is $0.51 \mathrm{mg} / \mathrm{ml}$ and $\mathrm{F} 6$ is four folds more, i.e., $4.36 \mathrm{mg} / \mathrm{ml}$ than active drug. From Figs. 1 and 2, surface morphology and shape were visualized by SEM. The particles were appeared as spheres with smooth surface and wavy.

The average particle size was found to be in a nanorange, i.e., $<1 \mu \mathrm{m}$ in the range of 150-350 nm, from this study, it has been concluded that there is a size reduction of particles, and from Figs. 3 and 4, it was confirmed

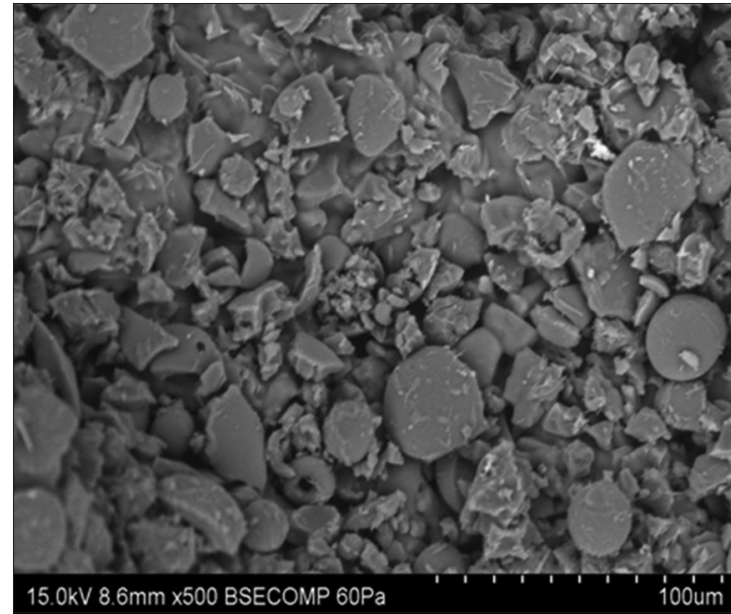

Fig. 1: Pictogram of surface morphology and spherical nanoparticles of itraconazole

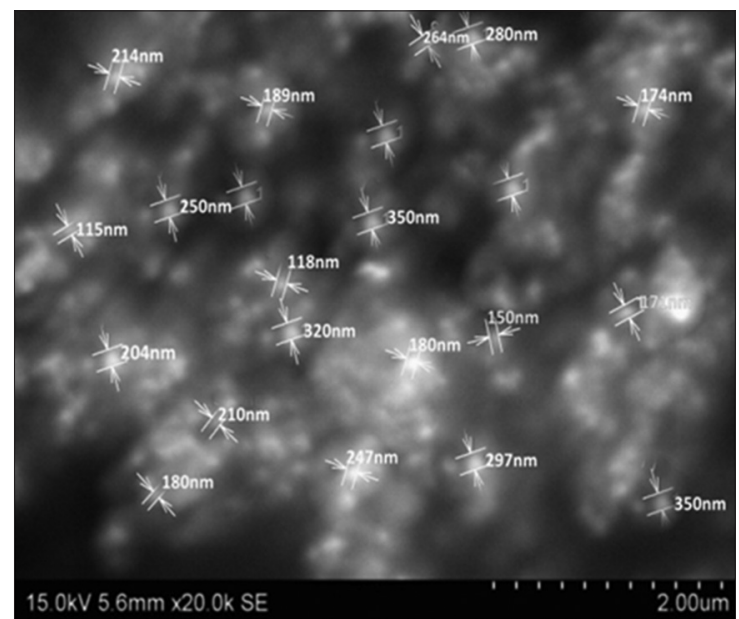

Fig. 2: Pictogram of itraconazole nanosuspension particles in nanorange

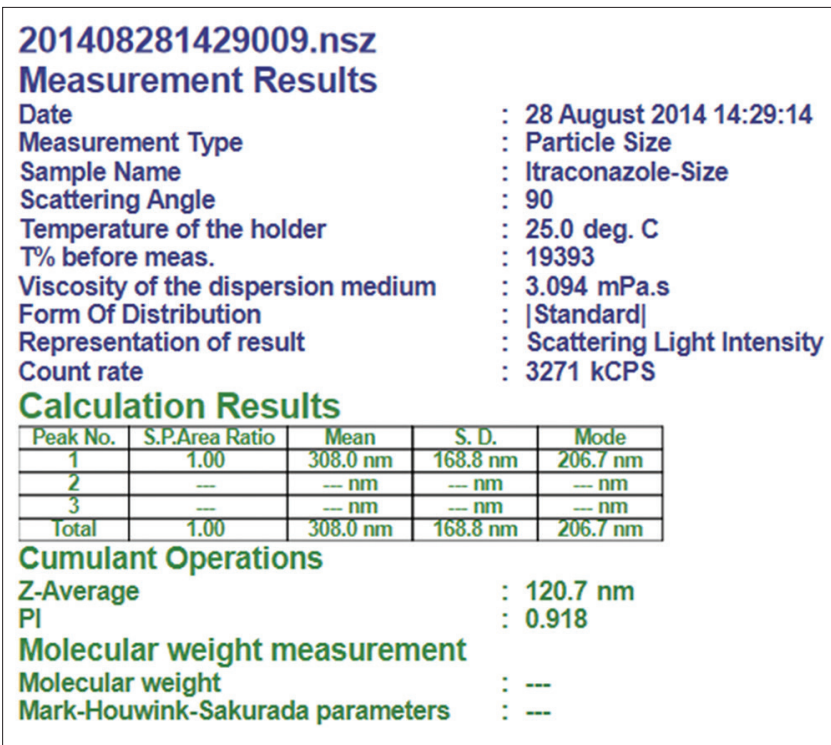

Fig. 3: Zeta size of itraconazole nanosuspension 


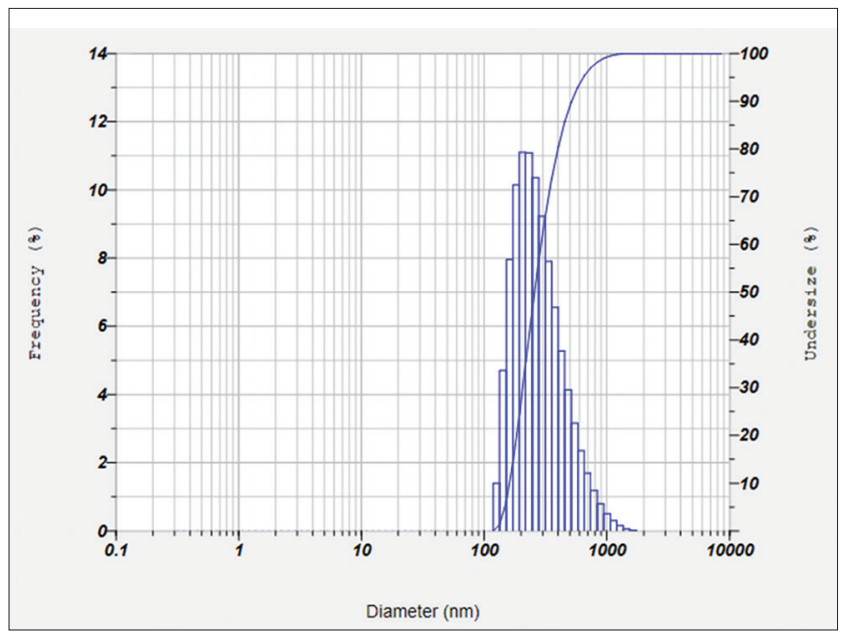

Fig. 4: Zeta size of itraconazole nanosuspension

\section{Measurement Results}

\section{Itraconazole-Zeta.nzt Measurement Results}

\section{Date}

Measurement Type

Sample Name

28 August 2014 14:41:54

Itraconazole-Zeta

Temperature of the holder $\quad: 25.0 \mathrm{deg}$. C

Viscosity of the dispersion medium : $0.894 \mathrm{mPa}$.s

Conductivity

$0.117 \mathrm{mS} / \mathrm{cm}$

Electrode Voltage

$3.4 \mathrm{~V}$

Calculation Results

\begin{tabular}{l|l|l|l|}
\hline Peak No. & Zeta Potential & Electrophoretic Mobility \\
\hline
\end{tabular}

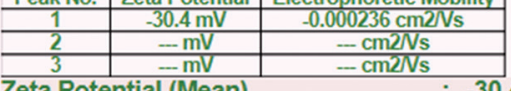

Zeta Potential (Mean) : $-30.4 \mathrm{mV}$

Electrophoretic Mobility mean $\quad:-0.000236 \mathrm{~cm} 2 / \mathrm{Vs}$

Fig. 5: Zeta potential of itraconazole nanosuspension

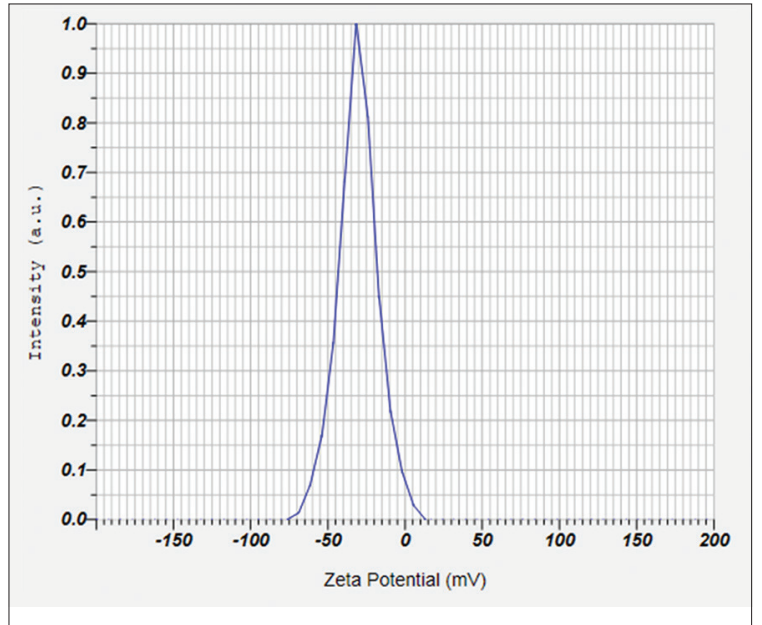

Fig. 6: Zeta potential of itraconazole nanosuspension

by zeta size by zeta sizer is determined as $308 \mathrm{~nm}$. From optical microscopy, SEM, and zeta potential, it was concluded that all particles fall in nanorange and the suspensions were nanosuspensions. The zeta potential was used to predict the storage stability of dispersed systems.

From the Figs 5 and 6 , zeta potential was found to be $-30.4 \pm 2.84 \mathrm{mV}$ for nanosuspension, and it indicates that there is physical stability due to sufficient thickness of diffusive layer to prevent particle aggregation and

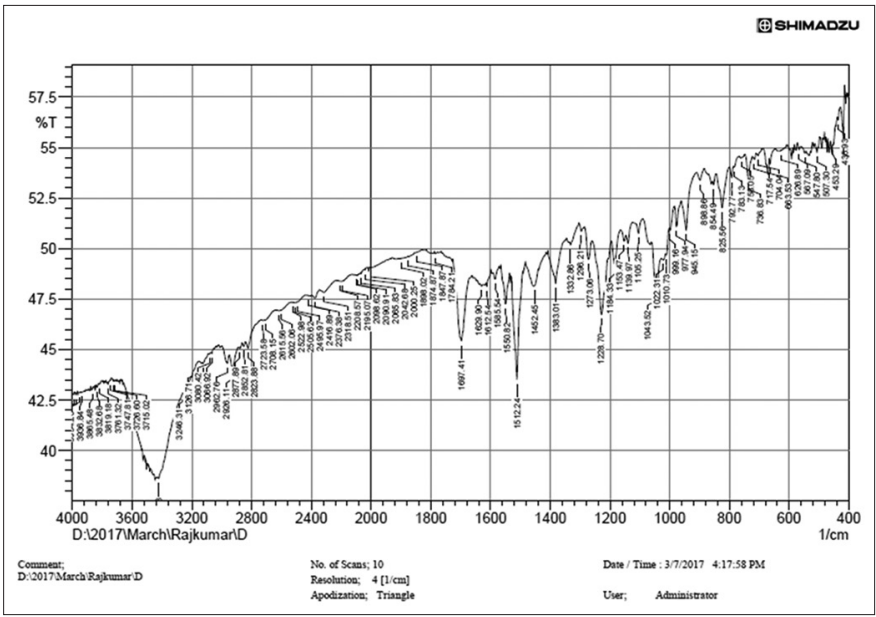

Fig. 7: Fourier-transform infrared spectroscopy spectra of pure drug - itraconazole

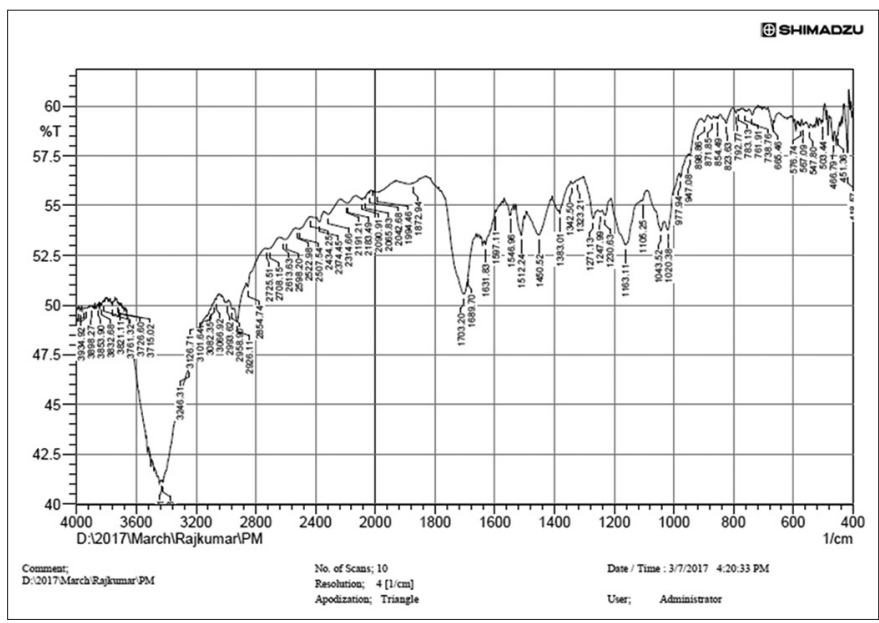

Fig. 8: Fourier-transform infrared spectroscopy spectra of physical mixture

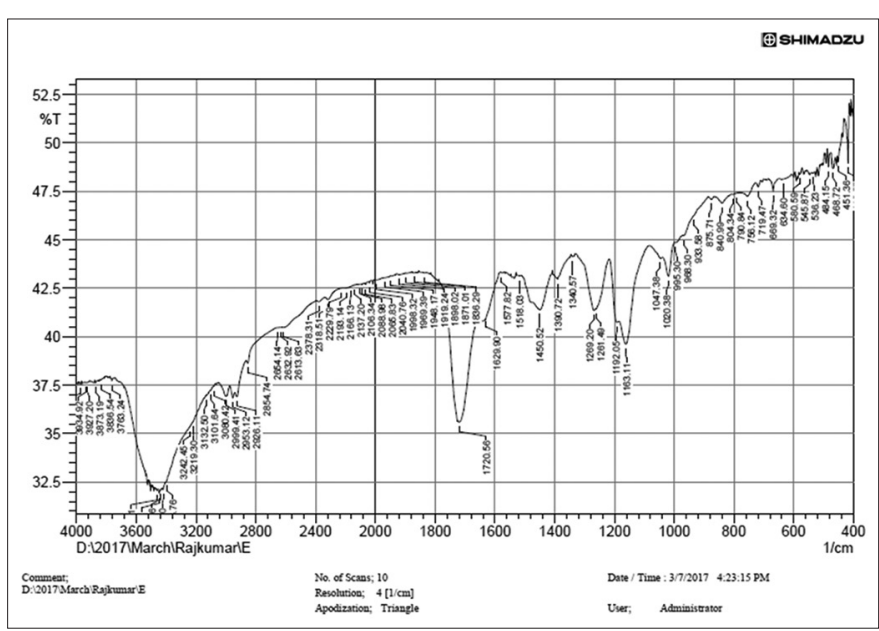

Fig. 9: Fourier-transform infrared spectroscopy spectra of itraconazole nanosuspension

allow for a longer residence time for the particles by ionic interaction with the drug molecules due to presences of quaternary ammonium groups of Eudragit RL100.

From the Figs 7-9, FTIR spectra of pure drug, physical mixture, and optimized formulation F6, it was observed that all characteristic peaks 
of itraconazole were present in the combination spectrum and there is no shift in peaks, thus indicating compatibility of the itraconazole with polymers where there is no physical and chemical interaction of drug and polymers. Thus, it confirms drug and excipients are compatible.

From the Fig. 10, XRPD spectra showed sharp, distinct peaks at $18.2^{\circ}$, $23.6^{\circ}, 30.0^{\circ}, 37.6^{\circ}, 33.3^{\circ}$, and $43.8^{\circ}$ confirm that it is in the crystalline

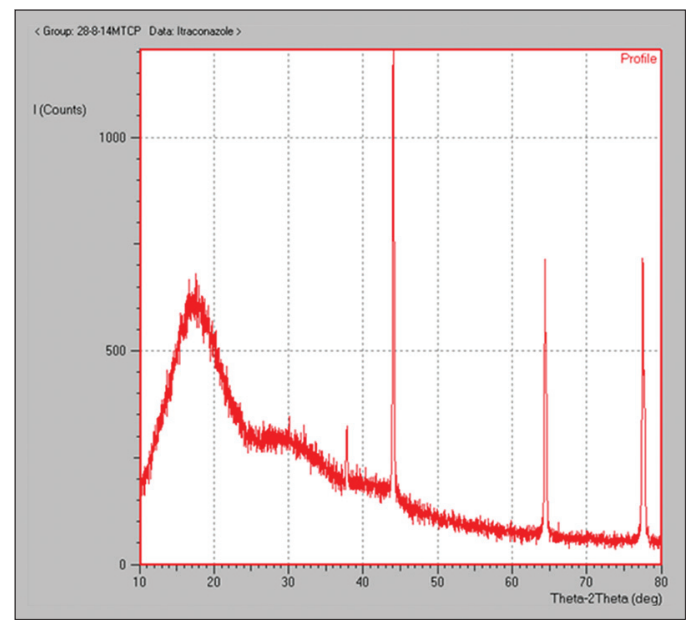

Fig. 10: X-ray powder diffraction spectra of pure drug

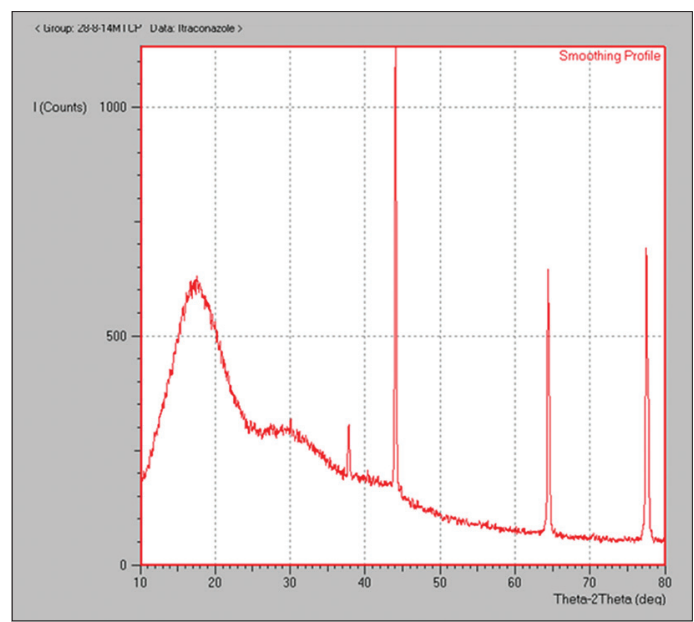

Fig. 11: X-ray powder diffraction spectra of itraconazole nanosuspension state of itraconazole. The X-ray diffraction of optimized nanosuspension F6 formulation as shown in Fig. 11 indicates the absence of itraconazole constructive peaks and points out that itraconazole is converted to amorphous state due to amorphous nanosuspension. The peaks obtained for pure drug were very clear and sharp, and the intensity of the peaks was very high when compared with peaks of nebivolol hydrochloride nanosuspensions. Reduction in the peak intensity indicates amorphization. From this, we can conclude that there was reduction in the crystallanity and change into amorphous structures. It was observed that the crystallanity of the drug was changed in the nanosuspensions. The peaks obtained for pure drug was very clear and sharp, and the intensity of the peaks was very high when compared to peaks of Itraconazolenanosuspensions. Reduction in the peak intensity indicates the change in crystal structure. From this, we can conclude that there was a reduction in the crystallanity and change into amorphous structures. The physical state of itraconazole in the polymer would also influence the release characteristics of drug and it was determined by DSC studies.

To probe this effect, DSC analysis was performed on itraconazole and optimized nanosuspension F6. From the DSC thermograms as shown in Figs 12 and 13, it was observed that characteristic thermogram of drug at $68.6^{\circ} \mathrm{C}$, but in thermogram of optimized formulation $\mathrm{F} 6$, the thermogram disappeared at $68.6^{\circ} \mathrm{C}$ and thermogram shown at $148.6^{\circ} \mathrm{C}$. Hence, it indicates that the physical nature of drug is changed in the formulation, the crystalline form of drug is changed to amorphous form DSC results, and it was confirmed as amorphous nanosuspension.

The results of dissolution studies were obtained from Table 2 and Fig. 14, revealing that the onset of dissolution of pure itraconazole was very low about $34.46 \%$ in $1 \mathrm{~h}$. It clearly indicates that the $\%$ drug release for pure itraconazole is very low, and nanosizing of drugs can lead to a dramatic increase in their oral absorption and subsequently bioavailability. From the data obtained, it was observed that there was an increase in \% drug release of $96.77 \%$ in $1 \mathrm{~h}$ for the itraconazole nanosuspension prepared by solvent displacement/nanoprecipitation method, respectively. It clearly indicates that nanosuspension formulation has been successful technique to improve the dissolution rate, thereby enhancing bioavailability. The amelioration in oral bioavailability can be attributed to the adhesiveness of the drug nanosuspension, increased surface area due to reduction in particle size, increased saturation solubility, leading to an increased concentration gradient between the gastrointestinal tract lumen and blood, and increased dissolution velocity.

\section{CONCLUSION}

Stable itraconazole nanosuspensions were successfully prepared and these nanosuspensions demonstrated dramatic improvement in dissolution rate of the active drug. Therefore, it is concluded that

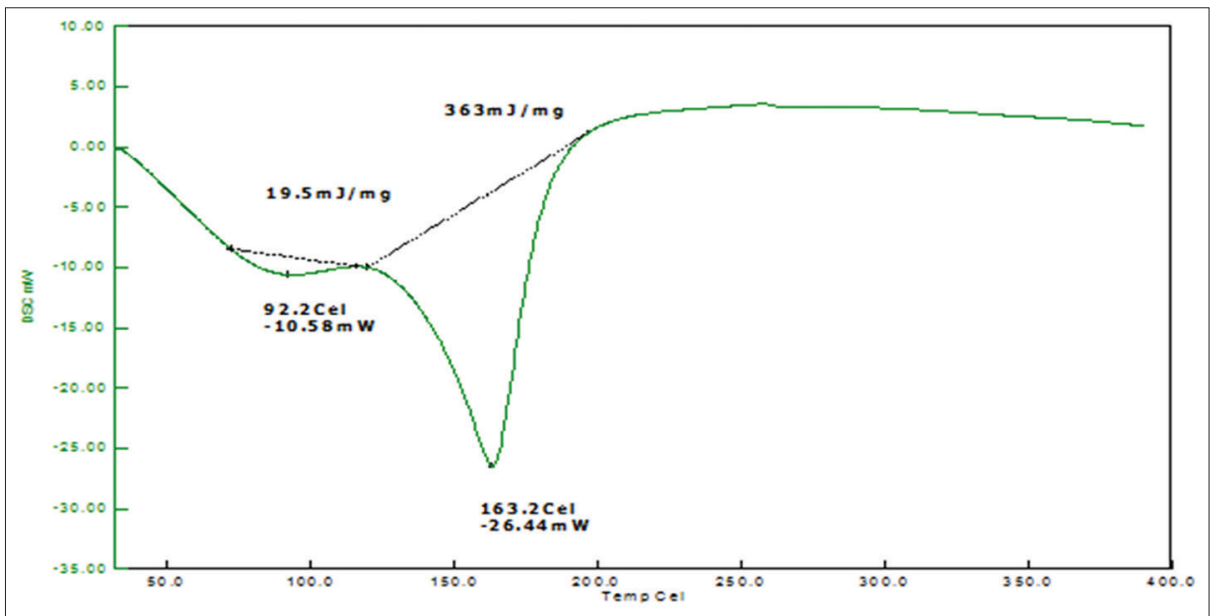

Fig. 12: Differential scanning calorimetry thermogram of pure drug - itraconazole 
Table 2: Dissolution profile of itraconazole nanosuspensions prepared by nanoprecipitation method

\begin{tabular}{|c|c|c|c|c|c|c|c|}
\hline Time in min & Pure drug & F1 & F2 & F3 & F4 & F5 & F6 \\
\hline 0 & 0 & 0 & 0 & 0 & 0 & 0 & 0 \\
\hline 5 & 10.25 & 58.48 & 61.85 & 63.78 & 53.84 & 69.28 & 72.53 \\
\hline 10 & 16.23 & 60.12 & 63.51 & 65.28 & 58.21 & 72.54 & 75.6 \\
\hline 15 & 18.32 & 65.85 & 68.64 & 69.12 & 62.58 & 75.48 & 80.79 \\
\hline 20 & 22.35 & 69.51 & 72.52 & 73.65 & 68.42 & 78.85 & 86.71 \\
\hline 30 & 26.32 & 70.34 & 75.80 & 78.89 & 70.56 & 81.54 & 89.01 \\
\hline 45 & 30.35 & 72.40 & 79.56 & 82.67 & 74.96 & 84.69 & 92.58 \\
\hline
\end{tabular}

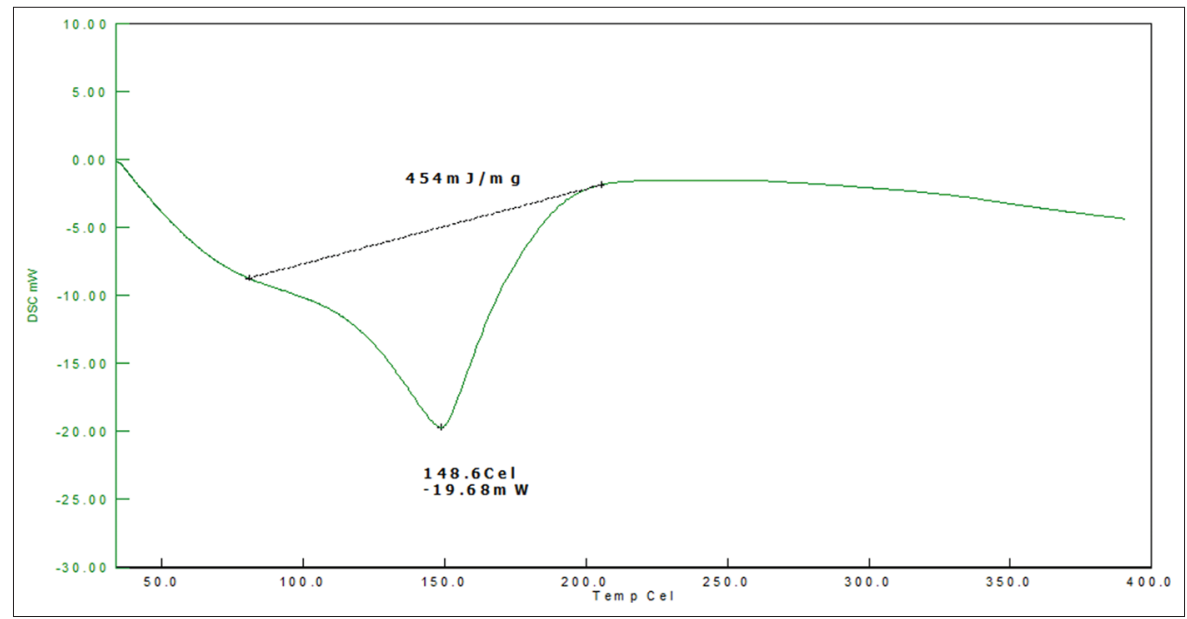

Fig. 13: Differential scanning calorimetry thermogram of itraconazole nanosuspension

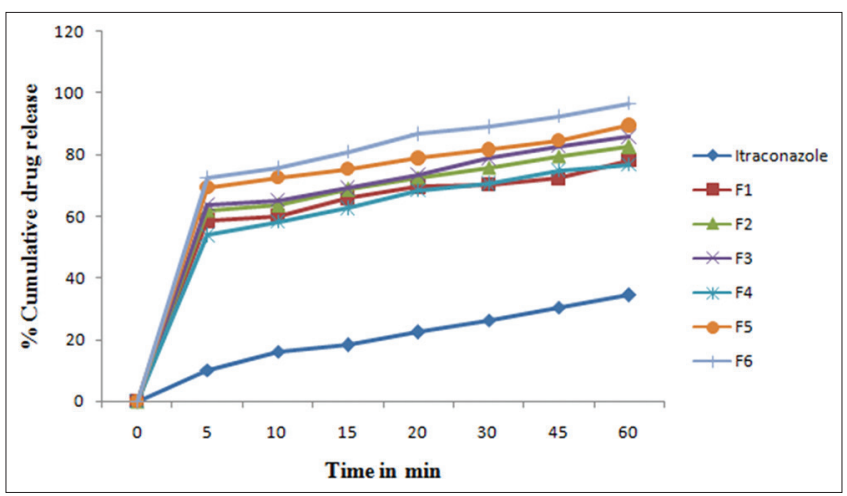

Fig. 14: Dissolution profile of itraconazole nanosuspensions

the nanoprecipitation method employed for the preparation of nanosuspension significantly improved dissolution rate and showed good evaluation studies of the nanosuspension formulation.

\section{ACKNOWLEDGMENTS}

The authors would like to acknowledge the management of Mother Teresa College of Pharmacy for providing necessary support to the conduction of this work. Furthermore, the authors would like to acknowledge Department of Technology, Osmania University, Hyderabad, for providing analytical support to this project.

\section{REFERENCES}

1. Nallaguntla L, Muzib YI, Aukunuru J, Balekari U. Novel nanoparticles for oral delivery of low molecular weight heparin: In vitro and in vivo assessment. Asian J Pharm Clin Res 2017;10:254-61.

2. Hiendrawan S, Hartanti AW, Veriansyah B, Widjojosusumo E, Tjandrawinata RR. Solubility enhancement of ketoconazole via salt and cocrystal formation. Int J Pharm Pharm Sci 2015;7:160-4.
3. Pragna S, Mandowaraa VK, Deepak G, Shetulv P. Formulation of curcuminoid loaded solid lipid nanoparticles in order to improve oral bioavailability. Int J Pharm Pharm Sci 2015;7:278-82.

4. Thadkala K, Nanam PK, Rambabu B, Sailu C, Aukunuru J. Preparation and characterization of amorphous ezetimibe nanosuspensions intended for enhancement of oral bioavailability. Int J Pharm Investig 2014;4:131-7.

5. Nanam PK, Thadkala K, Chinta S, Aukunuru J. Investigation of various practical techniques to enhance dissolution of ezetimibe from oral tablets. J Young Pharm 2014;6:8-15.

6. Yi Y, Tu L, Hu K, Wu W, Feng J. The construction of peurarin nanocrystals and its pharmacokinetic and in vivo-in vitro correlation (IVIVC) studies on beagle dog. Coll Surf B Biointerf 2015;133:164-70.

7. Ho C, Davies AM, Sangha RS, Lau D, Lara P Jr., Chew HK, et al. Phase I/II trial of pemetrexed plus nab-paclitaxel in advanced solid tumor patients with emphasis on non-small cell lung cancer. Invest New Drugs 2013;31:1587-91.

8. Kompella UB, Bandi N, Ayalasomayajula SP. Subconjunctival nano- and microparticles sustain retinal delivery of budesonide, a corticosteroid capable of inhibiting VEGF expression. Invest Ophthalmol Vis Sci 2003;44:1192-201

9. Kolluru LP, Rizvi SA, D'Souza M, D'Souza MJ. Formulation development of albumin based theragnostic nanoparticles as a potential delivery system for tumor targeting. J Drug Target 2013;21:77-86.

10. Raju A, Reddy AJ, Satheesh J, Jithan AV. Preparation and characterization of nevirapine oral nanosuspensions. Indian J Pharm Sci 2014;76:62-72

11. Amidon GL, Lennernas H, Shah VP, Crison JR. A theoretical basis for a bio-pharmaceutic drug classification: The correlation of in-vitro drug product dissolution and in-vivo bioavailablility. Pharm Res 1995;12:413-20.

12. Yohei K. Formulation design for poorly water soluble drugs based on bio-pharmaceutics classification system. Int J Pharm Sci 2011;420:1-10.

13. Liversidge GG, Higak K, Kimura T. Effect of particle size reduction on dissolution and oral absorption of a poorly water-soluble drug cilostazol in beagle dogs. J Control Release 2006;111:56-64.

14. Yellela S, Krishniah R. Pharmaceutical technologies for enhancing oral bioavailability of poorly soluble drugs. J Bioequiv Availab 
2010;2:28-36

15. Hsu CH, Cui Z, Mumper RJ, Jay M. Micellarsolubilization of some poorly soluble antidiabetic drugs. AAPS Pharm Sci Technol 2010;9:939-43.

16. Chiba Y, Kohri N, Iseki K, Miyazaki K. Improvement of dissolution and bioavailability for mebendazole, an agent for human echinococcosis, by preparing solid dispersion with polyethylene glycol. Chem Pharm Bull (Tokyo) 1991;39:2158-60.

17. Desai PP. Overcoming poor oral bioavailability using nanoparticle formulations. Drug Discov Today Technol 2012;9:87-95.

18. Müller RH, Jacobs C, Kayser O. Nanosuspensions as particulate drug formulations in therapy. Rationale for development and what we can expect for the future. Adv Drug Deliv Rev 2001;47:3-19.

19. Hetal P, Vishnu P, SnehaP. Nanosuspensions of olmesartanmedoxmil for bioavailability enhancement. J Pharma Bioallied Sci 2011;3:426-34.

20. Ana M, Marco M, Bruno G. Miconazole nanosuspensions. Int J Pharm 2010;396:210-3

21. Kocbek P, Baumgartner S, Kristl J. Preparation and evaluation of nanosuspensions for enhancing the dissolution of poorly soluble drugs. Int J Pharm 2006;312:179-86.

22. Vishal R, Agarwal YK. Nanosuspension: An approach to enhance solubility of drugs. J Adv Pharm Tech Res 2011;2:81-7.

23. Odds FC, Oris M, Van Dorsselaer P, Van Gerven F. Activities of an intravenous formulation of itraconazole in experimental disseminated aspergillus, candida, and cryptococcus infections. Antimicrob Agents Chemother 2000;44:3180-3.

24. Xu Y, Liuc X, Liana R, Zheng S. Enhanced dissolution and oral bioavailability of aripiprazole nanosuspensions prepared by nanoprecipitation/homogenization based on acid-base neutralization. Int J Pharm 2012;438:287-95.

25. Mansour M, Pouretedal HR, Vida V. Preparation and characterization of ibuprofen nanoparticles by using solvent-anti-solvent precipitation. Open Conf Proc J 2011;2:88-94.

26. Kakran M, Sahoo NG, Li L, Judeh Z, Wang Y, Chong K, et al. Fabrication of drug nanoparticles by evaporative precipitation of nanosuspension. Int J Pharm 2010;383:285-92.

27. Xia D, Quan P, Piao H, Piao H, Sun S. Preparation of stable nitrindepine nano suspensions using precipitation-ultrasonication method for enhancement of dissolution of oral bioavailability. Adv Drug Deliv Rev 2011;47:19-25. 